\title{
CALCULATIONS ON SOME SEQUENCE SPACES
}

\section{BRUNO DE MALAFOSSE}

\author{
Received 16 September 2002
}

We deal with space of sequences generalizing the well-known spaces $w_{\infty}^{p}(\lambda), c_{\infty}(\lambda, \mu)$, replacing the operators $C(\lambda)$ and $\Delta(\mu)$ by their transposes. We get generalizations of results concerning the strong matrix domain of an infinite matrix $A$.

2000 Mathematics Subject Classification: 46A45, $40 \mathrm{C} 05$.

1. Notations and preliminary results. For a given infinite matrix $A=\left(a_{n m}\right)_{n, m \geq 1}$, the operators $A_{n}$ are defined, for any integer $n \geq 1$, by

$$
A_{n}(X)=\sum_{m=1}^{\infty} a_{n m} x_{m},
$$

where $X=\left(x_{n}\right)_{n \geq 1}$, the series intervening in the second member being convergent. So we are led to the study of the infinite linear system

$$
A_{n}(X)=b_{n}, \quad n=1,2, \ldots,
$$

where $B=\left(b_{n}\right)_{n \geq 1}$ is a one-column matrix and $X$ the unknown, see $[1,2,3,4,5,6,7$, 8, 10]. Equation (1.2) can be written in the form $A X=B$, where $A X=\left(A_{n}(X)\right)_{n \geq 1}$. In this paper, we will also consider $A$ an operator from a sequence space into another sequence space.

A Banach space $E$ of complex sequences with the norm \|\|$_{E}$ is a BK space if each projection $P_{n} X=x_{n}$ is continuous for all $X \in E$. A BK space $E$ is said to have AK, (see $[12,13]$ ), if $B=\sum_{m=1}^{\infty} b_{m} e_{m}$, for every $B=\left(b_{n}\right)_{n \geq 1} \in E$, (with $e_{n}=(0, \ldots, 1, \ldots), 1$ being in the $n$th position), that is,

$$
\left\|\sum_{m=N+1}^{\infty} b_{m} e_{m}\right\|_{E} \longrightarrow 0 \quad(n \longrightarrow \infty) .
$$

We will write $s$ for the set of all complex sequences, $l_{\infty}, c$, $c_{0}$ for the sets of bounded, convergent, and null sequences, respectively. We will denote by $c s$ and $l_{1}$ the sets of convergent and absolutely convergent series, respectively.

In all that follows we will use the set

$$
U^{+*}=\left\{\left(u_{n}\right)_{n \geq 1} \in s \mid u_{n}>0 \forall n\right\}
$$


From Wilansky's notations [15], we define for any sequence

$$
\alpha=\left(\alpha_{n}\right)_{n \geq 1} \in U^{+*},
$$

and for any set of sequences $E$, the set

$$
\left(\frac{1}{\alpha}\right)^{-1} * E=\left\{\left(x_{n}\right)_{n \geq 1} \in s \mid\left(\frac{x_{n}}{\alpha_{n}}\right)_{n} \in E\right\} .
$$

We will write $\alpha * E$ instead of $(1 / \alpha)^{-1} * E$ for short. So we get

$$
\alpha * E= \begin{cases}s_{\alpha}^{\circ} & \text { if } E=c_{0}, \\ s_{\alpha}^{(c)} & \text { if } E=c, \\ s_{\alpha} & \text { if } E=l_{\infty} .\end{cases}
$$

We have for instance

$$
\alpha * c_{0}=s_{\alpha}^{\circ}=\left\{\left(x_{n}\right)_{n \geq 1} \in s \mid x_{n}=o\left(\alpha_{n}\right) n \longrightarrow \infty\right\}
$$

Each of the spaces $\alpha * E$, where $E \in\left\{c_{0}, c, l_{\infty}\right\}$, is a BK space normed by

$$
\|X\|_{s_{\alpha}}=\sup _{n \geq 1}\left(\frac{\left|x_{n}\right|}{\alpha_{n}}\right)
$$

and $s_{\alpha}^{\circ}$ has AK.

Now let $\alpha=\left(\alpha_{n}\right)_{n \geq 1}$ and $\beta=\left(\beta_{n}\right)_{n \geq 1} \in U^{+*} . S_{\alpha, \beta}$ is the set of infinite matrices $A=\left(a_{n m}\right)_{n, m \geq 1}$ such that

$$
\left(a_{n m} \alpha_{m}\right)_{m \geq 1} \in l^{1} \quad \forall n \geq 1, \quad \sum_{m=1}^{\infty}\left(\left|a_{n m}\right| \alpha_{m}\right)=O\left(\beta_{n}\right) \quad(n \longrightarrow \infty)
$$

$S_{\alpha, \beta}$ is a Banach space with the norm

$$
\|A\|_{S_{\alpha, \beta}}=\sup _{n \geq 1}\left(\sum_{m=1}^{\infty}\left|a_{n m}\right| \frac{\alpha_{m}}{\beta_{n}}\right)
$$

Let $E$ and $F$ be any subsets of $s$. When $A$ maps $E$ into $F$, we will write $A \in(E, F)$, see [11]. So for every $X \in E, A X \in F,(A X \in F$ will mean that for each $n \geq 1$ the series defined by $y_{n}=\sum_{m=1}^{\infty} a_{n m} x_{m}$ is convergent and $\left.\left(y_{n}\right)_{n \geq 1} \in F\right)$. It has been proved in [9] that $A \in\left(s_{\alpha}, s_{\beta}\right)$ if and only if $A \in S_{\alpha, \beta}$. So we can write that $\left(s_{\alpha}, s_{\beta}\right)=S_{\alpha, \beta}$.

When $s_{\alpha}=s_{\beta}$, we obtain the unital Banach algebra $S_{\alpha, \beta}=S_{\alpha}$, (see $\left.[1,2,3,5,6,10]\right)$ normed by $\|A\|_{S_{\alpha}}=\|A\|_{S_{\alpha, \alpha}}$.

We also have $A \in\left(s_{\alpha}, s_{\alpha}\right)$ if and only if $A \in S_{\alpha}$. If $\|I-A\|_{S_{\alpha}}<1$, we will say that $A \in \Gamma_{\alpha}$. Since the set $S_{\alpha}$ is a unital algebra, we have the useful result that if $A \in \Gamma_{\alpha}, A$ is bijective from $s_{\alpha}$ into itself. 
If $\alpha=\left(r^{n}\right)_{n \geq 1}, \Gamma_{\alpha}, S_{\alpha}, s_{\alpha}, s_{\alpha}^{\circ}$, and $s_{\alpha}^{(c)}$ are replaced by $\Gamma_{r}, S_{r}, s_{r}, s_{r}^{\circ}$, and $s_{r}^{(c)}$, respectively, (see $[1,2,3,5,6,10]$ ). When $r=1$, we obtain $s_{1}=l_{\infty}, s_{1}^{\circ}=c_{0}$, and $s_{1}^{(c)}=c$, and putting $e=(1,1, \ldots)$, we have $S_{1}=S_{e}$. It is well known, see [11], that

$$
\left(s_{1}, s_{1}\right)=\left(c_{0}, s_{1}\right)=\left(c, s_{1}\right)=S_{1} .
$$

For any subset $E$ of $s$, we put

$$
A E=\{Y \in s \mid \exists X \in E, Y=A X\} .
$$

If $F$ is a subset of $s$, we will denote

$$
F(A)=F_{A}=\{X \in s \mid Y=A X \in F\}
$$

We can see that $F(A)=A^{-1} F$.

2. Some properties of the operators $\Delta^{+}$and $\Sigma^{+}$. Here we will deal with the operators represented by $C^{+}(\lambda)$ and $\Delta^{+}(\lambda)$.

Let

$$
U=\left\{\left(u_{n}\right)_{n \geq 1} \in s \mid u_{n} \neq 0 \forall n\right\}
$$

We define $C(\lambda)=\left(c_{n m}\right)_{n, m \geq 1}$, for $\lambda=\left(\lambda_{n}\right)_{n \geq 1} \in U$, by

$$
c_{n m}= \begin{cases}\frac{1}{\lambda_{n}} & \text { if } m \leq n \\ 0 & \text { otherwise }\end{cases}
$$

So, we put $C^{+}(\lambda)=C(\lambda)^{t}$. It can be proved that the matrix $\Delta(\lambda)=\left(c_{n m}^{\prime}\right)_{n, m \geq 1}$ with

$$
c_{n m}^{\prime}= \begin{cases}\lambda_{n} & \text { if } m=n \\ -\lambda_{n-1} & \text { if } m=n-1, n \geq 2 \\ 0 & \text { otherwise }\end{cases}
$$

is the inverse of $C(\lambda)$, see $[12,14]$. Similarly, we put $\Delta^{+}(\lambda)=\Delta(\lambda)^{t}$. If $\lambda=e$, we get the well-known operator of first difference represented by $\Delta(e)=\Delta$ and it is usually written as $\Sigma=C(e)$. Note that $\Delta=\Sigma^{-1}$ and $\Sigma$ belong to any given space $S_{R}$ with $R>1$. Writing $D_{\lambda}=\left(\lambda_{n} \delta_{n m}\right)_{n, m \geq 1}$, (where $\delta_{n m}=0$ for $n \neq m$ and $\delta_{n n}=1$ otherwise), we have $\Delta^{+}(\lambda)=D_{\lambda} \Delta^{+}$. So for any given $\alpha \in U^{+*}$, we see that if $\left(\alpha_{n-1} / \alpha_{n}\right)\left|\lambda_{n} / \lambda_{n-1}\right|=O(1)$, then $\Delta^{+}(\lambda) \in\left(s_{(\alpha /|\lambda|)}, s_{\alpha}\right)$. Since $\operatorname{Ker} \Delta^{+}(\lambda) \neq 0$, we are lead to define the set

$$
s_{\alpha}^{*}\left(\Delta^{+}(\lambda)\right)=s_{\alpha}\left(\Delta^{+}(\lambda)\right) \bigcap s_{(\alpha /|\lambda|)}=\left\{X=\left(x_{n}\right)_{n \geq 1} \in s_{(\alpha /|\lambda|)} \mid \Delta^{+}(\lambda) X \in s_{\alpha}\right\} .
$$


It can easily be seen that

$$
s_{(\alpha /|\lambda|)}^{*}\left(\Delta^{+}(e)\right)=s_{(\alpha /|\lambda|)}^{*}\left(\Delta^{+}\right)=s_{\alpha}^{*}\left(\Delta^{+}(\lambda)\right) .
$$

2.1. Properties of the sequence $C(\alpha) \alpha$. We will use the following sets:

$$
\begin{aligned}
\widehat{C_{1}} & =\left\{\alpha \in U^{+*} \mid \frac{1}{\alpha_{n}}\left(\sum_{k=1}^{n} \alpha_{k}\right)=O(1)(n \rightarrow \infty)\right\}, \\
\hat{C} & =\left\{\alpha \in U^{+*} \mid \frac{1}{\alpha_{n}}\left(\sum_{k=1}^{n} \alpha_{k}\right) \in c\right\}, \\
\widehat{C_{1}^{+}} & =\left\{\alpha \in U^{+*} \bigcap c s \mid \frac{1}{\alpha_{n}}\left(\sum_{k=n}^{\infty} \alpha_{k}\right)=O(1)(n \rightarrow \infty)\right\}, \\
\Gamma & =\left\{\alpha \in U^{+*} \mid \lim _{n \rightarrow \infty}\left(\frac{\alpha_{n-1}}{\alpha_{n}}\right)<1\right\}, \\
\Gamma^{+} & =\left\{\alpha \in U^{+*} \mid \overline{\lim _{n \rightarrow \infty}}\left(\frac{\alpha_{n+1}}{\alpha_{n}}\right)<1\right\} .
\end{aligned}
$$

Note that $\alpha \in \Gamma^{+}$if and only if $1 / \alpha \in \Gamma$. We will see in Proposition 2.1 that if $\alpha \in \widehat{C_{1}}, \alpha$ tends to infinity. On the other hand, we see that $\Delta \in \Gamma_{\alpha}$ implies $\alpha \in \Gamma$ and $\alpha \in \Gamma$ if and only if there is an integer $q \geq 1$ such that

$$
\gamma_{q}(\alpha)=\sup _{n \geq q+1}\left(\frac{\alpha_{n-1}}{\alpha_{n}}\right)<1
$$

We obtain the following results in which we put $[C(\alpha) \alpha]_{n}=\left(\sum_{k=1}^{n} \alpha_{k}\right) / \alpha_{n}$.

Proposition 2.1. Let $\alpha \in U^{+*}$. Then

(i) $\alpha_{n-1} / \alpha_{n} \rightarrow 0$ if and only if $[C(\alpha) \alpha]_{n} \rightarrow 1$,

(ii) (a) $\alpha \in \hat{C}$ implies that $\left(\alpha_{n-1} / \alpha_{n}\right)_{n \geq 1} \in c$,

(b) $[C(\alpha) \alpha]_{n} \rightarrow l$ implies that $\alpha_{n-1} / \alpha_{n} \rightarrow 1-1 / l$,

(iii) if $\alpha \in \widehat{C_{1}}$, there are $K>0$ and $\gamma>1$ such that

$$
\alpha_{n} \geq K \gamma^{n} \quad \forall n
$$

(iv) the condition $\alpha \in \Gamma$ implies that $\alpha \in \widehat{C_{1}}$ and there exists a real $b>0$ such that

$$
\left.[C(\alpha) \alpha]_{n} \leq \frac{1}{1-\chi}+b \chi^{n} \quad \text { for } n \geq q+1, \chi=\gamma_{q}(\alpha) \in\right] 0,1[
$$

(v) the condition $\alpha \in \Gamma^{+}$implies that $\alpha \in \widehat{C_{1}^{+}}$. 
Proof. Assume that $\alpha_{n-1} / \alpha_{n} \rightarrow 0$. Then there is an integer $N$ such that

$$
n \geq N+1 \Longrightarrow \frac{\alpha_{n-1}}{\alpha_{n}} \leq \frac{1}{2}
$$

So there exists a real $K>0$ such that $\alpha_{n} \geq K 2^{n}$ for all $n$ and

$$
\frac{\alpha_{k}}{\alpha_{n}}=\frac{\alpha_{k}}{\alpha_{k+1}} \cdots \frac{\alpha_{n-1}}{\alpha_{n}} \leq\left(\frac{1}{2}\right)^{n-k} \text { for } N \leq k \leq n-1
$$

Then

$$
\frac{1}{\alpha_{n}}\left(\sum_{k=1}^{n-1} \alpha_{k}\right)=\frac{1}{\alpha_{n}}\left(\sum_{k=1}^{N-1} \alpha_{k}\right)+\sum_{k=N}^{n-1} \frac{\alpha_{k}}{\alpha_{n}} \leq \frac{1}{K 2^{n}}\left(\sum_{k=1}^{N-1} \alpha_{k}\right)+\sum_{k=N}^{n-1}\left(\frac{1}{2}\right)^{n-k},
$$

and since $\sum_{k=N}^{n-1}(1 / 2)^{n-k}=1-(1 / 2)^{n-N} \rightarrow 1,(n \rightarrow \infty)$, we deduce that

$$
\frac{1}{\alpha_{n}}\left(\sum_{k=1}^{n-1} \alpha_{k}\right)=O(1)
$$

and $\left([C(\alpha) \alpha]_{n}\right) \in l_{\infty}$. Using the identity

$$
[C(\alpha) \alpha]_{n}=\frac{\alpha_{1}+\cdots+\alpha_{n-1}}{\alpha_{n-1}} \frac{\alpha_{n-1}}{\alpha_{n}}+1=[C(\alpha) \alpha]_{n-1}\left(\frac{\alpha_{n-1}}{\alpha_{n}}\right)+1,
$$

we get $[C(\alpha) \alpha]_{n} \rightarrow 1$. This proves the necessity.

Conversely, if $[C(\alpha) \alpha]_{n} \rightarrow 1$, then

$$
\frac{\alpha_{n-1}}{\alpha_{n}}=\frac{[C(\alpha) \alpha]_{n}-1}{[C(\alpha) \alpha]_{n-1}} \longrightarrow 0
$$

(ii) is a direct consequence of the identity (2.14).

(iii) We put $\Sigma_{n}=\sum_{k=1}^{n} \alpha_{k}$. Then for a real $M>1$,

$$
[C(\alpha) \alpha]_{n}=\frac{\Sigma_{n}}{\sum_{n}-\Sigma_{n-1}} \leq M \quad \forall n
$$

So $\Sigma_{n} \geq(M /(M-1)) \Sigma_{n-1}$ and $\Sigma_{n} \geq(M /(M-1))^{n-1} \alpha_{1} \forall n$. Therefore, from

$$
\frac{\alpha_{1}}{\alpha_{n}}\left(\frac{M}{M-1}\right)^{n-1} \leq[C(\alpha) \alpha]_{n}=\frac{\Sigma_{n}}{\alpha_{n}} \leq M
$$

we conclude that $\alpha_{n} \geq K \gamma^{n}$ for all $n$, with $K=(M-1) \alpha_{1} / M^{2}$ and $\gamma=M /(M-1)>1$. 
(iv) If $\alpha \in \Gamma$, there is an integer $q \geq 1$ for which

$$
k \geq q+1 \text { implies } \frac{\alpha_{k-1}}{\alpha_{k}} \leq \chi<1 \text {, with } \chi=\gamma_{q}(\alpha) \text {. }
$$

So there is a real $M^{\prime}>0$ for which

$$
\alpha_{n} \geq \frac{M^{\prime}}{\chi^{n}} \quad \forall n \geq q+1
$$

Writing $\sigma_{n q}=\left(1 / \alpha_{n}\right)\left(\sum_{k=1}^{q} \alpha_{k}\right)$ and $d_{n}=[C(\alpha) \alpha]_{n}-\sigma_{n q}$, we get

$$
d_{n}=\frac{1}{\alpha_{n}}\left(\sum_{k=q+1}^{n} \alpha_{k}\right)=1+\sum_{j=q+1}^{n-1}\left(\prod_{k=1}^{n-j} \frac{\alpha_{n-k}}{\alpha_{n-k+1}}\right) \leq \sum_{j=q+1}^{n} x^{n-j} \leq \frac{1}{1-\chi} .
$$

Using (2.19), we get $\sigma_{n q} \leq\left(1 / M^{\prime}\right) \chi^{n}\left(\sum_{k=1}^{q} \alpha_{k}\right)$. So

$$
[C(\alpha) \alpha]_{n} \leq a+b x^{n}
$$

with $a=1 /(1-\chi)$ and $b=\left(1 / M^{\prime}\right)\left(\sum_{k=1}^{q} \alpha_{k}\right)$.

(v) If $\alpha \in \Gamma^{+}$, there are $\left.\chi^{\prime} \in\right] 0,1\left[\right.$ and an integer $q^{\prime} \geq 1$ such that

$$
\frac{\alpha_{k}}{\alpha_{k-1}} \leq \chi^{\prime} \quad \text { for } k \geq q^{\prime} .
$$

Then for every $n \geq q^{\prime}$, we have

$$
\frac{1}{\alpha_{n}}\left(\sum_{k=n}^{\infty} \alpha_{k}\right)=\sum_{k=n}^{\infty}\left(\frac{\alpha_{k}}{\alpha_{n}}\right) \leq 1+\sum_{k=n+1}^{\infty} \prod_{i=0}^{k-n-1}\left(\frac{\alpha_{k-i}}{\alpha_{k-i-1}}\right) \leq \sum_{k=n}^{\infty} \chi^{\prime k-n}=O(1) .
$$

This gives the conclusion.

REMARK 2.2. Note that as a direct consequence of Proposition 2.1, we have $\widehat{C_{1}} \cap \widehat{C_{1}^{+}}=$ $\Gamma \cap \Gamma^{+}=\phi$.

REMARK 2.3. The condition $\alpha \in \widehat{C_{1}}$ does not imply that $\alpha \in \Gamma$, see [8].

2.2. Some new properties of the operators $\Delta$ and $\Delta^{+}$. In the following we will use some lemmas, the next one is well known, see [15].

LEMMA 2.4. The condition $A \in\left(c_{0}, c_{0}\right)$ is equivalent to

$$
\begin{gathered}
A \in S_{1}, \\
\lim _{n} a_{n m}=0 \quad \text { for each } m \geq 1 .
\end{gathered}
$$

LEMMA 2.5. If $\Delta^{+}$is bijective from $s_{\alpha}$ into itself, then $\alpha \in c s$.

Proof. Assume that $\alpha \notin c s$, that is, $\sum_{n} \alpha_{n}=\infty$. Two cases are possible.

(1) $e \in \operatorname{Ker} \Delta^{+} \cap s_{\alpha}$. Then $\Delta^{+}$cannot be bijective from $s_{\alpha}$ into itself.

(2) $e \notin \operatorname{Ker} \Delta^{+} \bigcap s_{\alpha}$. Then $1 / \alpha \notin s_{1}$ and there is a sequence of integers $\left(n_{i}\right)_{i}$ strictly increasing such that $1 / \alpha_{n_{i}} \rightarrow \infty$. Assume that the equation $\Delta^{+} X=\alpha$ has a solution 
$X=\left(x_{n, 0}\right)_{n \geq 1}$ in $s_{\alpha}$. Then there is a unique scalar $x_{1}$ such that

$$
x_{n, 0}=x_{1}-\sum_{k=1}^{n-1} \alpha_{k}
$$

So

$$
\frac{\left|x_{n_{i}, 0}\right|}{\alpha_{n_{i}}}=\left|\frac{1}{\alpha_{n_{i}}}\left(x_{1}-\sum_{k=1}^{n_{i}-1} \alpha_{k}\right)\right| \rightarrow \infty \quad \text { as } i \rightarrow \infty
$$

and $X \notin s_{\alpha}$, which is contradictory.

We conclude that each of the properties $e \in \operatorname{Ker} \Delta^{+} \cap s_{\alpha}$ and $e \notin \operatorname{Ker} \Delta^{+} \cap s_{\alpha}$ is impossible and $\Delta^{+}$is not bijective from $s_{\alpha}$ into itself. This proves the lemma.

LEMMA 2.6. For every $X \in c_{0}, \Sigma^{+}\left(\Delta^{+} X\right)=X$ and for every $X \in c s, \Delta^{+}\left(\Sigma^{+} X\right)=X$.

Proof. It can easily be seen that

$$
\begin{aligned}
& {\left[\Sigma^{+}\left(\Delta^{+} X\right)\right]_{n}=\sum_{m=n}^{\infty}\left(x_{m}-x_{m+1}\right)=x_{n} \quad \forall X \in c_{0},} \\
& {\left[\Delta^{+}\left(\Sigma^{+} X\right)\right]_{n}=\sum_{m=n}^{\infty} x_{m}-\sum_{m=n+1}^{\infty} x_{m}=x_{n} \quad \forall X \in c s .}
\end{aligned}
$$

We can assert the following result, in which we put $\alpha^{+}=\left(\alpha_{n+1}\right)_{n \geq 1}$ and $s_{\alpha}^{\circ *}\left(\Delta^{+}\right)=$ $s_{\alpha}^{\circ}\left(\Delta^{+}\right) \bigcap s_{\alpha}^{\circ}$. Note that from (2.5) we have

$$
s_{\alpha}^{*}\left(\Delta^{+}(e)\right)=s_{\alpha}^{*}\left(\Delta^{+}\right)=s_{\alpha}\left(\Delta^{+}\right) \bigcap s_{\alpha} .
$$

THEOREM 2.7. (i) (a) $s_{\alpha}(\Delta)=s_{\alpha}$ if and only if $\alpha \in \widehat{C_{1}}$,

(b) $s_{\alpha}^{\circ}(\Delta)=s_{\alpha}^{\circ}$ if and only if $\alpha \in \widehat{C_{1}}$,

(c) $s_{\alpha}^{(c)}(\Delta)=s_{\alpha}^{(c)}$ if and only if $\alpha \in \hat{C}$.

(ii) (a) $\alpha \in \widehat{C_{1}}$ if and only if $s_{\alpha^{+}}\left(\Delta^{+}\right)=s_{\alpha}$ and $\Delta^{+}$is surjective from $s_{\alpha}$ into $s_{\alpha^{+}}$,

(b) $\alpha \in \widehat{C_{1}^{+}}$if and only if $s_{\alpha}^{*}\left(\Delta^{+}\right)=s_{\alpha}$ and $\Delta^{+}$is bijective from $s_{\alpha}$ into $s_{\alpha}$,

(c) $\alpha \in \widehat{C_{1}^{+}}$implies that $s_{\alpha}^{\circ *}\left(\Delta^{+}\right)=s_{\alpha}^{\circ}$ and $\Delta^{+}$is bijective from $s_{\alpha}^{\circ}$ into $s_{\alpha}^{\circ}$.

(iii) $\alpha \in \widehat{C_{1}^{+}}$if and only if $s_{\alpha}\left(\Sigma^{+}\right)=s_{\alpha}$ and $s_{\alpha}\left(\Sigma^{+}\right)=s_{\alpha}$ implies $s_{\alpha}^{\circ}\left(\Sigma^{+}\right)=s_{\alpha}^{\circ}$.

Proof. (i) has been proved in [8].

(ii)(a) Sufficiency. If $\Delta^{+}$is surjective from $s_{\alpha}$ into $s_{\alpha^{+}}$, then for every $B \in s_{\alpha^{+}}$the solutions of $\Delta^{+} X=B$ in $s_{\alpha}$ are given by

$$
x_{n+1}=x_{1}-\sum_{k=1}^{n} b_{k} \quad n=1,2, \ldots
$$


where $x_{1}$ is arbitrary. If we take $B=\alpha^{+}$, we get $x_{n}=x_{1}-\sum_{k=2}^{n} \alpha_{k}$. So

$$
\frac{x_{n}}{\alpha_{n}}=\frac{x_{1}}{\alpha_{n}}-\frac{1}{\alpha_{n}}\left(\sum_{k=2}^{n} \alpha_{k}\right)=O(1)
$$

Taking $x_{1}=-\alpha_{1}$, we conclude that $\left(\sum_{k=1}^{n-1} \alpha_{k}\right) / \alpha_{n}=O(1)$ and $\alpha \in \widehat{C_{1}}$.

Conversely, assume that $\alpha \in \widehat{C_{1}}$. From the inequality

$$
\frac{\alpha_{n-1}}{\alpha_{n}} \leq \frac{1}{\alpha_{n}}\left(\sum_{k=1}^{n} \alpha_{k}\right)=O(1)
$$

we deduce that $\alpha_{n-1} / \alpha_{n}=O(1)$ and $\Delta^{+} \in\left(s_{\alpha}, s_{\alpha^{+}}\right)$. Then for any given $B \in s_{\alpha^{+}}$, the solutions of the equation $\Delta^{+} X=B$ are given by $x_{1}=-u$ and

$$
-x_{n}=u+\sum_{k=1}^{n-1} b_{k} \text { for } n \geq 2
$$

where $u$ is an arbitrary scalar. So there exists a real $K>0$ such that

$$
\frac{\left|x_{n}\right|}{\alpha_{n}}=\frac{\left|u+\sum_{k=1}^{n-1} b_{k}\right|}{\alpha_{n}} \leq \frac{|u|+K\left(\sum_{k=2}^{n} \alpha_{k}\right)}{\alpha_{n}}=O(1)
$$

and $X \in s_{\alpha}$. We conclude that $\Delta^{+}$is surjective from $s_{\alpha}$ into $s_{\alpha^{+}}$.

(ii)(b) Necessity. Assume that $\alpha \in \widehat{C_{1}^{+}}$. Then $\Delta^{+} \in\left(s_{\alpha}, s_{\alpha}\right)$, since

$$
\frac{\alpha_{n+1}}{\alpha_{n}} \leq \frac{1}{\alpha_{n}}\left(\sum_{k=n}^{\infty} \alpha_{k}\right)=O(1) \quad(n \longrightarrow \infty) .
$$

Further, from $s_{\alpha} \subset c s$, we deduce, using Lemma 2.4, that for any given $B \in s_{\alpha}$,

$$
\Delta^{+}\left(\Sigma^{+} B\right)=B
$$

On the other hand, $\Sigma^{+} B=\left(\sum_{k=n}^{\infty} b_{k}\right)_{n \geq 1} \in s_{\alpha}$, since $\alpha \in \widehat{C_{1}^{+}}$. So $\Delta^{+}$is surjective from $s_{\alpha}$ into $s_{\alpha}$. Finally, $\Delta^{+}$is injective because the equation

$$
\Delta^{+} X=O
$$

admits the unique solution $X=O$ in $s_{\alpha}$, since

$$
\operatorname{Ker} \Delta^{+}=\left\{u e^{t} \mid u \in C\right\}
$$

and $e^{t} \notin s_{\alpha}$. 
Sufficiency. For every $B \in s_{\alpha}$, the equation $\Delta^{+} X=B$ admits a unique solution in $s_{\alpha}$. Then from Lemma 2.5, $\alpha \in c s$ and since $s_{\alpha} \subset c s$, we deduce from Lemma 2.6 that $X=\Sigma^{+} B \in s_{\alpha}$ is the unique solution of $\Delta^{+} X=B$. Taking $B=\alpha$, we get $\Sigma^{+} \alpha \in s_{\alpha}$, that is, $\alpha \in \widehat{C_{1}^{+}}$.

(ii)(c) If $\alpha \in \widehat{C_{1}^{+}}, \Delta^{+}$is bijective from $s_{\alpha}^{\circ}$ into itself. Indeed, we have $D_{1 / \alpha} \Delta^{+} D_{\alpha} \in\left(c_{0}, c_{0}\right)$ from (2.34) and Lemma 2.4. Furthermore, since $\alpha \in \widehat{C_{1}^{+}}$we have $s_{\alpha}^{\circ} \subset c s$ and for every $B \in s_{\alpha}^{\circ}$,

$$
\Delta^{+}\left(\Sigma^{+} B\right)=B
$$

From Lemma 2.4, we have $\Sigma^{+} \in\left(s_{\alpha}^{\circ}, s_{\alpha}^{\circ}\right)$, so the equation $\Delta^{+} X=B$ admits the solution $X_{0}=\Sigma^{+} B$ in $s_{\alpha}^{\circ}$ and we have proved that $\Delta^{+}$is surjective from $s_{\alpha}^{\circ}$ into itself. Finally, $\alpha \in \widehat{C_{1}^{+}}$implies that $e^{t} \notin s_{\alpha}^{\circ}$, so Ker $\Delta^{+} \bigcap s_{\alpha}^{\circ}=\{0\}$ and we conclude that $\Delta^{+}$is bijective from $s_{\alpha}^{\circ}$ into itself.

(iii) comes from (ii), since $\alpha \in \widehat{C_{1}^{+}}$if and only if $\Delta^{+}$is bijective from $s_{\alpha}$ into itself and

$$
\Sigma^{+}\left(\Delta^{+} X\right)=\Delta^{+}\left(\Sigma^{+} X\right)=X \quad \forall X \in s_{\alpha}
$$

As a direct consequence of Theorem 2.7 we obtain the following results.

COROLLARY 2.8. Let $R$ be any real $>0$. Then

$$
R>1 \Longleftrightarrow s_{R}(\Delta)=s_{R} \Longleftrightarrow s_{R}^{\circ}(\Delta)=s_{R}^{\circ} \Longleftrightarrow s_{R}\left(\Delta^{+}\right)=s_{R}
$$

Proof. From (i) and (ii) in Theorem 2.7, we see that it is enough to prove that $\alpha=$ $\left(R^{n}\right)_{n \geq 1} \in \widehat{C_{1}}$ if and only if $R>1$. We have $\left(R^{n}\right)_{n \geq 1} \in \widehat{C_{1}}$ if and only if $R \neq 1$ and

$$
R^{-n}\left(\sum_{k=1}^{n} R^{k}\right)=\frac{1}{1-R} R^{-n+1}-\frac{R}{1-R}=O(1) \quad \text { as } n \rightarrow \infty .
$$

This means that $R>1$ and the corollary is proved.

Using the notation $\alpha^{-}=\left(1, \alpha_{1}, \alpha_{2}, \ldots, \alpha_{n-1}, \ldots\right)$ we get the next result.

Corollary 2.9. Let $\alpha \in U^{+*}$ and $\mu \in U$. Then

(i) $\alpha /|\mu| \in \widehat{C_{1}}$ if and only if

$$
s_{\alpha}\left(\Delta^{+}(\mu)\right)=s_{(\alpha /|\mu|)^{-}},
$$

(ii) $\alpha /|\mu| \in \widehat{C_{1}^{+}}$if and only if

$$
s_{\alpha}^{*}\left(\Delta^{+}(\mu)\right)=s_{(\alpha /|\mu|)} .
$$

Proof. First we have

$$
s_{\alpha}\left(\Delta^{+}(\mu)\right)=s_{(\alpha /|\mu|)}\left(\Delta^{+}\right) .
$$


Indeed,

$$
X \in s_{\alpha}\left(\Delta^{+}(\mu)\right) \Longleftrightarrow D_{\mu} \Delta^{+} X \in s_{\alpha} \Longleftrightarrow \Delta^{+} X \in s_{(\alpha /|\mu|)} \Longleftrightarrow X \in s_{(\alpha /|\mu|)}\left(\Delta^{+}\right) .
$$

Now, if $\alpha /|\mu| \in \widehat{C_{1}}$, from (i) in Theorem 2.7, we have $s_{(\alpha /|\mu|)}\left(\Delta^{+}\right)=s_{(\alpha /|\mu|)^{-}}$and $s_{\alpha}\left(\Delta^{+}(\mu)\right)=s_{(\alpha /|\mu|)^{-}}$. Conversely, assume $s_{\alpha}\left(\Delta^{+}(\mu)\right)=s_{(\alpha /|\mu|)^{-}}$. Reasoning as above, we get $s_{(\alpha /|\mu|)}\left(\Delta^{+}\right)=s_{(\alpha /|\mu|)^{-}}$, and using (i) in Theorem 2.7 we conclude that $\alpha /|\mu| \in \widehat{C_{1}}$ and (i) holds.

(ii) $\alpha /|\mu| \in \widehat{C_{1}^{+}}$implies that $\Delta^{+}$is bijective from $s_{(\alpha /|\mu|)}$ into itself. Thus

$$
s_{\alpha}^{*}\left(\Delta^{+}(\mu)\right)=s_{(\alpha /|\mu|)}^{*}\left(\Delta^{+}\right)=s_{(\alpha /|\mu|)} .
$$

This proves the necessity. Conversely, assume that $s_{\alpha}^{*}\left(\Delta^{+}(\mu)\right)=s_{(\alpha /|\mu|)}$. Then $s_{(\alpha /|\mu|)}^{*}\left(\Delta^{+}\right)$ $=s_{(\alpha /|\mu|)}$ and from Theorem 2.7(ii)(b), $\alpha /|\mu| \in \widehat{C_{1}^{+}}$and (ii) holds.

2.3. Spaces $w_{\alpha}^{p}(\lambda)$ and $w_{\alpha}^{+p}(\lambda)$ for given real $p>0$. Here we will define sets generalizing the well-known sets

$$
\begin{aligned}
& w_{\infty}^{p}(\lambda)=\left\{X \in s \mid C(\lambda)\left(|X|^{p}\right) \in l_{\infty}\right\}, \\
& w_{0}^{p}(\lambda)=\left\{X \in s \mid C(\lambda)\left(|X|^{p}\right) \in c_{0}\right\},
\end{aligned}
$$

see $[9,12,13,14,15]$. It is proved that each of the sets $w_{0}^{p}=w_{0}^{p}\left((n)_{n}\right)$ and $w_{\infty}^{p}=$ $w_{\infty}^{p}\left((n)_{n}\right)$ is a $p$-normed FK space for $0<p<1$ (i.e., a complete linear metric space for which each projection $P_{n}$ is continuous) and a BK space for $1 \leq p<\infty$ with respect to the norm

$$
\|X\|= \begin{cases}\sup _{v \geq 1}\left(\frac{1}{2^{v}}\left(\sum_{n=2^{v}}^{2^{v+1}-1}\left|x_{n}\right|^{p}\right)\right) & \text { if } 0<p<1, \\ \sup _{v \geq 1}\left(\frac{1}{2^{v}}\left(\sum_{n=2^{v}}^{2^{v+1}-1}\left|x_{n}\right|^{p}\right)\right)^{1 / p} & \text { if } 1 \leq p<\infty .\end{cases}
$$

The set $w_{0}^{p}$ has the property $\mathrm{AK}$, (i.e., every $X=\left(x_{n}\right)_{n \geq 1} \in w_{0}^{p}$ has a unique representation $\left.X=\sum_{n=1}^{\infty} x_{n} e_{n}^{t}\right)$ and every sequence $X=\left(x_{n}\right)_{n \geq 1} \in w^{p}$ has a unique representation

$$
X=l e^{t}+\sum_{n=1}^{\infty}\left(x_{n}-l\right) e_{n}^{t},
$$

where $l \in C$ is such that $X-l e^{t} \in w_{0}^{p}$, (see [4]). Now, let $\alpha \in U^{+*}$ and $\lambda \in U^{+*}$. We have

$$
\begin{aligned}
w_{\alpha}^{p}(\lambda) & =\left\{X \in s \mid C(\lambda)\left(|X|^{p}\right) \in s_{\alpha}\right\}, \\
w_{\alpha}^{+p}(\lambda) & =\left\{X \in s \mid C^{+}(\lambda)\left(|X|^{p}\right) \in s_{\alpha}\right\}, \\
w_{\alpha}^{\circ p}(\lambda) & =\left\{X \in s \mid C(\lambda)\left(|X|^{p}\right) \in s_{\alpha}^{\circ}\right\}, \\
w_{\alpha}^{\circ+p}(\lambda) & =\left\{X \in s \mid C^{+}(\lambda)\left(|X|^{p}\right) \in s_{\alpha}^{\circ}\right\} .
\end{aligned}
$$

We deduce from the previous section the following theorem. 
THEOREM 2.10. (i) (a) The condition $\alpha \in \widehat{C_{1}^{+}}$is equivalent to

$$
w_{\alpha}^{+p}(\lambda)=s_{(\alpha \lambda)^{1 / p}}
$$

(b) If $\alpha \in \widehat{C_{1}^{+}}$, then

$$
w_{\alpha}^{\circ p}(\lambda)=s_{(\alpha \lambda) 1 / p}^{\circ}
$$

(ii) (a) The condition $\alpha \lambda \in \widehat{C_{1}}$ is equivalent to

$$
w_{\alpha}^{p}(\lambda)=s_{(\alpha \lambda)^{1 / p}} .
$$

(b) If $\alpha \lambda \in \widehat{C_{1}}$, then

$$
w_{\alpha}^{\circ+p}(\lambda)=s_{(\alpha \lambda)^{1 / p}}^{\circ}
$$

Proof. Assume that $\alpha \in \widehat{C_{1}^{+}}$. Since $C^{+}(\lambda)=\Sigma^{+} D_{1 / \lambda}$, we have

$$
w_{\alpha}^{+p}(\lambda)=\left\{X \mid\left(\Sigma^{+} D_{1 / \lambda}\right)\left(|X|^{p}\right) \in s_{\alpha}\right\}=\left\{X \mid D_{1 / \lambda}\left(|X|^{p}\right) \in s_{\alpha}\left(\Sigma^{+}\right)\right\}
$$

and since $\alpha \in \widehat{C_{1}^{+}}$implies $s_{\alpha}\left(\Sigma^{+}\right)=s_{\alpha}$, we conclude that

$$
w_{\alpha}^{+p}(\lambda)=\left\{\left.X|| X\right|^{p} \in D_{\lambda} s_{\alpha}=s_{\alpha \lambda}\right\}=s_{(\alpha \lambda)^{1 / p}} .
$$

Conversely, we have $(\alpha \lambda)^{1 / p} \in s_{(\alpha \lambda)^{1 / p}}=w_{\alpha}^{+p}(\lambda)$. So

$$
C^{+}(\lambda)\left[(\alpha \lambda)^{1 / p}\right]^{p}=\left(\sum_{k=n}^{\infty} \frac{\alpha_{k} \lambda_{k}}{\lambda_{k}}\right)_{n \geq 1} \in s_{\alpha},
$$

that is, $\alpha \in \widehat{C_{1}^{+}}$and we have proved (i). We obtain (i)(b) by reasoning as above.

(ii) Assume that $\alpha \lambda \in \widehat{C_{1}}$. Then

$$
w_{\alpha}^{p}(\lambda)=\left\{\left.X|| X\right|^{p} \in \Delta(\lambda) s_{\alpha}\right\} .
$$


Since $\Delta(\lambda)=\Delta D_{\lambda}$, we get $\Delta(\lambda) s_{\alpha}=\Delta s_{\alpha \lambda}$. Now, from $\alpha \lambda \in \widehat{C_{1}}$ we deduce that $\Delta$ is bijective from $s_{\alpha \lambda}$ into itself and $w_{\alpha}^{p}(\lambda)=s_{(\alpha \lambda)^{1 / p}}$. Conversely, assume that $w_{\alpha}^{p}(\lambda)=$ $s_{(\alpha \lambda)^{1 / p}}$. Then $(\alpha \lambda)^{1 / p} \in s_{(\alpha \lambda)^{1 / p}}$ implies that

$$
C(\lambda)(\alpha \lambda) \in s_{\alpha}
$$

and since $D_{1 / \alpha} C(\lambda)(\alpha \lambda) \in s_{1}=l_{\infty}$, we conclude that $C(\alpha \lambda)(\alpha \lambda) \in l_{\infty}$. The proof of (ii)(b) follows the same lines as in the proof of the necessity in (ii) replacing $s_{\alpha \lambda}$ by $s_{\alpha \lambda}^{\circ}$.

3. New sets of sequences of the form $\left[A_{1}, A_{2}\right]$. In this section, we will deal with the sets

$$
\left[A_{1}(\lambda), A_{2}(\mu)\right]=\left\{X \in s \mid A_{1}(\lambda)\left(\left|A_{2}(\mu) X\right|\right) \in s_{\alpha}\right\}
$$

where $A_{1}$ and $A_{2}$ are of the form $C(\xi), C^{+}(\xi), \Delta(\xi)$, or $\Delta^{+}(\xi)$ and we give necessary conditions to get $\left[A_{1}(\lambda), A_{2}(\mu)\right]$ in the form $s_{\gamma}$.

Let $\lambda$ and $\mu \in U^{+*}$. For simplification, we will write throughout this section

$$
\left[A_{1}, A_{2}\right]=\left[A_{1}(\lambda), A_{2}(\mu)\right]=\left\{X \in s \mid A_{1}(\lambda)\left(\left|A_{2}(\mu) X\right|\right) \in s_{\alpha}\right\}
$$

for any matrices

$$
\begin{aligned}
& A_{1}(\lambda) \in\left\{\Delta(\lambda), \Delta^{+}(\lambda), C(\lambda), C^{+}(\lambda)\right\}, \\
& A_{2}(\mu) \in\left\{\Delta(\mu), \Delta^{+}(\mu), C(\mu), C^{+}(\mu)\right\} .
\end{aligned}
$$

So we have for instance

$$
[C, \Delta]=\left\{X \in s \mid C(\lambda)(|\Delta(\mu) X|) \in s_{\alpha}\right\}=\left(w_{\alpha}(\lambda)\right)_{\Delta(\mu)}, \ldots
$$

In all that follows, the conditions $\xi \in \Gamma$, or $1 / \eta \in \Gamma$ for any given sequences $\xi$ and $\eta$ can be replaced by the conditions $\xi \in \widehat{C_{1}}$ and $\eta \in \widehat{C_{1}^{+}}$.

3.1. Spaces $[C, C],[C, \Delta],[\Delta, C]$, and $[\Delta, \Delta]$. For the convenience of the reader we will write the following identities, where $A_{1}(\lambda)$ and $A_{2}(\mu)$ are lower triangles and we will use the convention $\mu_{0}=0$ :

$$
\begin{aligned}
& {[C, C]=\left\{X \in s \mid \frac{1}{\lambda_{n}}\left(\sum_{m=1}^{n}\left|\frac{1}{\mu_{m}}\left(\sum_{k=1}^{m} x_{k}\right)\right|\right)=\alpha_{n} O(1)\right\},} \\
& {[C, \Delta]=\left\{X \in s \mid \frac{1}{\lambda_{n}}\left(\sum_{k=1}^{n}\left|\mu_{k} x_{k}-\mu_{k-1} x_{k-1}\right|\right)=\alpha_{n} O(1)\right\},} \\
& {[\Delta, C]=\left\{X \in S\left|-\lambda_{n-1}\right| \frac{1}{\mu_{n-1}}\left(\sum_{k=1}^{n-1} x_{i}\right)\left|+\lambda_{n}\right| \frac{1}{\mu_{n}}\left(\sum_{k=1}^{n} x_{i}\right) \mid=\alpha_{n} O(1)\right\},} \\
& {[\Delta, \Delta]=\left\{X \in S\left|-\lambda_{n-1}\right| \mu_{n-1} x_{n-1}-\mu_{n-2} x_{n-2}\left|+\lambda_{n}\right| \mu_{n} x_{n}-\mu_{n-1} x_{n-1} \mid=\alpha_{n} O(1)\right\} .}
\end{aligned}
$$


Note that for $\alpha=e$ and $\lambda=\mu,[C, \Delta]$ is the well-known set of sequences that are strongly bounded, denoted by $c_{\infty}(\lambda)$, see $[9,12,13,14,15]$. We get the following result.

THEOREM 3.1. (i) If $\alpha \lambda$ and $\alpha \lambda \mu \in \Gamma$, then

$$
[C, C]=S_{(\alpha \lambda \mu)},
$$

(ii) if $\alpha \lambda \in \Gamma$, then

$$
[C, \Delta]=s_{(\alpha(\lambda / \mu))},
$$

(iii) if $\alpha$ and $\alpha \mu / \lambda \in \Gamma$, then

$$
[\Delta, C]=s_{(\alpha(\mu / \lambda))},
$$

(iv) if $\alpha$ and $\alpha / \lambda \in \Gamma$, then

$$
[\Delta, \Delta]=S_{(\alpha(\mu / \lambda))} .
$$

Proof. We have for any given $X$

$$
C(\lambda)(|C(\mu) X|) \in s_{\alpha}
$$

if and only if $C(\mu) X \in s_{\alpha}(C(\lambda))=s_{(\alpha \lambda)}$, since $\alpha \lambda \in \Gamma$. So we get

$$
X \in \Delta(\mu) s_{\alpha \lambda}
$$

and the condition $\alpha \lambda \mu \in \Gamma$ implies $\Delta(\mu) s_{\alpha \lambda}=s_{(\alpha \lambda \mu)}$, which permits us to conclude (i).

(ii) Now, for any given $X$, the condition $C(\lambda)(|\Delta(\mu) X|) \in s_{\alpha}$ is equivalent to

$$
|\Delta(\mu) X| \in \Delta(\lambda) s_{\alpha}=\Delta s_{\alpha \lambda}=s_{\alpha \lambda},
$$

since $\alpha \lambda \in \Gamma$. Thus

$$
X \in C(\mu) s_{\alpha \lambda}=D_{1 / \mu} \sum s_{\alpha \lambda}=s_{(\alpha(\lambda / \mu))} .
$$

(iii) Similarly, $\Delta(\lambda)(|C(\mu) X|) \in s_{\alpha}$ if and only if

$$
|C(\mu) X| \in s_{\alpha}(\Delta(\lambda))=C(\lambda) s_{\alpha}=D_{1 / \lambda} \Sigma s_{\alpha}=s_{(\alpha / \lambda)},
$$

since $\alpha \in \Gamma$. So

$$
X \in \Delta(\mu) s_{(\alpha / \lambda)}=\Delta s_{(\alpha \mu / \lambda)} .
$$

We conclude since $\alpha \mu / \lambda \in \Gamma$ implies that $\Delta s_{(\alpha \mu / \lambda)}=s_{(\alpha \mu / \lambda)}$. (iv) Here,

$$
\Delta(\lambda)(|\Delta(\mu) X|) \in s_{\alpha} \text { if and only if } \Delta(\mu) X \in C(\lambda) s_{\alpha}=s_{(\alpha / \lambda)},
$$

if $\alpha \in \Gamma$. Thus we have

$$
X \in C(\mu) s_{(\alpha / \lambda)}=s_{(\alpha / \lambda \mu)}
$$

since $\alpha / \lambda \in \Gamma$. So (iv) holds. 
REMARK 3.2. If we define

$$
\left[A_{1}, A_{2}\right]_{0}=\left\{X \in s \mid A_{1}(\lambda)\left(\left|A_{2}(\mu) X\right|\right) \in s_{\alpha}^{\circ}\right\}
$$

we get the same results as in Theorem 3.1, replacing in each case (i), (ii), (iii), and (iv) $s \xi$ by $s_{\xi}^{\circ}$.

3.2. Sets $\left[\Delta, \Delta^{+}\right],\left[\Delta, C^{+}\right],\left[C, \Delta^{+}\right],\left[\Delta^{+} \Delta\right],\left[\Delta^{+}, C\right],\left[\Delta^{+} \Delta^{+}\right],\left[C^{+}, C\right],\left[C^{+}, \Delta\right],\left[C^{+}, \Delta^{+}\right]$, and $\left[C^{+}, C^{+}\right]$. We get immediately from the definitions of the operators $\Delta(\xi), \Delta^{+}(\eta)$, $C(\xi)$, and $C^{+}(\eta)$, the following:

$$
\begin{aligned}
{\left[\Delta, \Delta^{+}\right] } & =\left\{X\left|\lambda_{n}\right| \mu_{n} x_{n}-\mu_{n+1} x_{n+1}\left|-\lambda_{n-1}\right| \mu_{n-1} x_{n-1}-\mu_{n} x_{n} \mid=\alpha_{n} O(1)\right\}, \\
{\left[\Delta, C^{+}\right] } & =\left\{X\left|\lambda_{n}\right| \sum_{i=n}^{\infty} \frac{x_{i}}{\mu_{i}}\left|-\lambda_{n-1}\right| \sum_{i=n-1}^{\infty} \frac{x_{i}}{\mu_{i}} \mid=\alpha_{n} O(1)\right\}, \\
{\left[C, \Delta^{+}\right] } & =\left\{X \mid \frac{1}{\lambda_{n}}\left(\sum_{k=1}^{n}\left|\mu_{k} x_{k}-\mu_{k+1} x_{k+1}\right|\right)=\alpha_{n} O(1)\right\}, \\
{\left[\Delta^{+}, \Delta\right] } & =\left\{X\left|\lambda_{n}\right| \mu_{n} x_{n}-\mu_{n-1} x_{n-1}\left|-\lambda_{n+1}\right| \mu_{n+1} x_{n+1}-\mu_{n} x_{n} \mid=\alpha_{n} O(1)\right\}, \\
{\left[\Delta^{+}, C\right] } & =\left\{X\left|\frac{\lambda_{n}}{\mu_{n}}\right| \sum_{i=1}^{n} x_{i}\left|-\frac{\lambda_{n+1}}{\mu_{n+1}}\right| \sum_{i=1}^{n+1} x_{i} \mid=\alpha_{n} O(1)\right\}, \\
{\left[\Delta^{+}, \Delta^{+}\right] } & =\left\{X\left|\lambda_{n}\right| \mu_{n} x_{n}-\mu_{n+1} x_{n+1}\left|-\lambda_{n+1}\right| \mu_{n+1} x_{n+1}-\mu_{n+2} x_{n+2} \mid=\alpha_{n} O(1)\right\}, \\
{\left[C^{+}, C\right] } & =\left\{X \mid \sum_{k=n}^{\infty}\left(\frac{1}{\lambda_{k}}\left|\frac{1}{\mu_{k}} \sum_{i=1}^{k} x_{i}\right|\right)=\alpha_{n} O(1)\right\}, \\
{\left[C^{+}, \Delta\right] } & =\left\{X \mid \sum_{k=n}^{\infty}\left(\frac{1}{\lambda_{k}}\left|\mu_{k} x_{k}-\mu_{k-1} x_{k-1}\right|\right)=\alpha_{n} O(1)\right\}, \\
{\left[C^{+}, \Delta^{+}\right] } & =\left\{X \mid \sum_{k=n}^{\infty}\left(\frac{1}{\lambda_{k}}\left|\mu_{k} x_{k}-\mu_{k+1} x_{k+1}\right|\right)=\alpha_{n} O(1)\right\}, \\
{\left[C^{+}, C^{+}\right] } & =\left\{X \mid \sum_{k=n}^{\infty}\left(\frac{1}{\lambda_{k}}\left|\sum_{i=k}^{\infty} \frac{x_{i}}{\mu_{i}}\right|\right)=\alpha_{n} O(1)\right\} .
\end{aligned}
$$

We can assert the following result, in which we do the convention $\alpha_{n}=1$ for $n \leq 0$.

THEOREM 3.3. (i) Assume that $\alpha \in \Gamma$. Then

$$
\begin{aligned}
& {\left[\Delta, \Delta^{+}\right]=s_{(\alpha / \lambda \mu)^{-}} \quad \text { if } \frac{\alpha}{\lambda \mu} \in \Gamma,} \\
& {\left[\Delta, C^{+}\right]=s_{(\alpha(\mu / \lambda))} \quad \text { if } \frac{\lambda}{\alpha} \in \Gamma .}
\end{aligned}
$$

(ii) The conditions $\alpha \lambda \in \Gamma$ and $\alpha \lambda / \mu \in \Gamma$ together imply

$$
\left[C, \Delta^{+}\right]=s_{(\alpha(\lambda / \mu))^{-}} .
$$


(iii) The condition $\alpha / \lambda \in \Gamma$ implies

$$
\left[\Delta^{+}, \Delta\right]=S_{\left(\alpha_{n-1} / \mu_{n} \lambda_{n-1}\right)_{n}}=S_{\left(1 / \mu(\alpha / \lambda)^{-}\right)} .
$$

(iv) If $\alpha / \lambda$ and $\mu(\alpha / \lambda)^{-}=\left(\mu_{n}\left(\alpha_{n-1} / \lambda_{n-1}\right)\right)_{n} \in \Gamma$, then

$$
\left[\Delta^{+}, C\right]=s_{\mu(\alpha / \lambda)^{-}} .
$$

(v) If $\alpha / \lambda$ and $1 / \mu(\alpha / \lambda)^{-}=\left(\alpha_{n-1} / \mu_{n} \lambda_{n-1}\right)_{n} \in \Gamma$, then

$$
\left[\Delta^{+}, \Delta^{+}\right]=s_{\left((\alpha / \lambda)^{-} / \mu\right)^{-}}=s_{\left(\alpha_{n-2} / \lambda_{n-2} \mu_{n-1}\right)_{n} .} .
$$

(vi) If $1 / \alpha$ and $\alpha \lambda \mu \in \Gamma$, then

$$
\left[C^{+}, C\right]=s_{(\alpha \lambda \mu)} .
$$

(vii) If $1 / \alpha$ and $\alpha \lambda \in \Gamma$, then

$$
\left[C^{+}, \Delta\right]=s_{(\alpha(\lambda / \mu))} .
$$

(viii) If $1 / \alpha$ and $\alpha(\lambda / \mu) \in \Gamma$, then

$$
\left[C^{+}, \Delta^{+}\right]=S_{(\alpha(\lambda / \mu))^{-}} .
$$

(ix) If $1 / \alpha$ and $1 / \alpha \lambda \in \Gamma$, then

$$
\left[C^{+}, C^{+}\right]=s_{(\alpha \lambda \mu)} .
$$

Proof. (i) First, for any given $X$, the condition $\Delta(\lambda)\left(\left|\Delta^{+}(\mu) X\right|\right) \in s_{\alpha}$ is equivalent to

$$
\left|\Delta^{+}(\mu) X\right| \in s_{\alpha}(\Delta(\lambda))=s_{(\alpha / \lambda)},
$$

since $\alpha \in \Gamma$. So $X \in s_{\alpha \lambda}\left(\Delta^{+}(\mu)\right)$ and applying Corollary 2.9, we conclude the first part of the proof of (i).

We have $\Delta(\lambda)\left(\left|C^{+}(\mu) X\right|\right) \in s_{\alpha}$ if and only if

$$
\left|C^{+}(\mu) X\right| \in C(\lambda) s_{\alpha}=D_{1 / \lambda} \Sigma s_{\alpha} .
$$

Since $\alpha \in \Gamma$, we have $\Sigma s_{\alpha}=s_{\alpha}$ and $D_{1 / \lambda} \Sigma s_{\alpha}=s_{(\alpha / \lambda)}$. Then, for $\alpha / \lambda \in \Gamma^{+}, X \in\left[\Delta, C^{+}\right]$if and only if

$$
X \in w_{(\alpha / \lambda)}^{+1}(\mu)=s_{(\alpha(\mu / \lambda))} .
$$

(ii) For any given $X, C(\lambda)\left(\left|\Delta^{+}(\mu) X\right|\right) \in s_{\alpha}$ is equivalent to

$$
\Delta^{+}(\mu) X \in w_{\alpha}^{1}(\lambda),
$$

and since $\alpha \lambda \in \Gamma$ we have $w_{\alpha}^{1}(\lambda)=s_{\alpha \lambda}$. So

$$
X \in s_{\alpha \lambda}\left(\Delta^{+}(\mu)\right)=s_{(\alpha(\lambda / \mu))^{-}}
$$

if $\alpha \lambda / \mu \in \Gamma$. Then (ii) is proved. 
(iii) Here, $\Delta^{+}(\lambda)(|\Delta(\mu) X|) \in s_{\alpha}$ if and only if

$$
|\Delta(\mu) X| \in s_{\alpha}\left(\Delta^{+}(\lambda)\right)=s_{(\alpha / \lambda)^{-}},
$$

since $\alpha / \lambda \in \Gamma$. Thus

$$
X \in C(\mu) s_{(\alpha / \lambda)^{-}}=D_{1 / \mu} \Sigma s_{(\alpha / \lambda)^{-}}=s_{\left(\alpha_{n-1} / \lambda_{n-1} \mu_{n}\right)}
$$

if $(\alpha / \lambda)^{-} \in \Gamma$, that is, $\alpha / \lambda \in \Gamma$.

(iv) If $\alpha / \lambda \in \Gamma$, we get

$$
\begin{aligned}
\Delta^{+}(\lambda)(|C(\mu) X|) & \in s_{\alpha} \Longleftrightarrow|C(\mu) X| \in s_{\alpha}\left(\Delta^{+}(\lambda)\right) \\
& =s_{(\alpha / \lambda)^{-}} \Longleftrightarrow X \in \Delta(\mu) s_{(\alpha / \lambda)^{-}} .
\end{aligned}
$$

Since $\mu(\alpha / \lambda)^{-} \in \Gamma$, we conclude that $\left[\Delta^{+}, C\right]=s_{\left(\mu(\alpha / \lambda)^{-}\right)}$.

(v) One has

$$
\left[\Delta^{+}, \Delta^{+}\right]=\left\{X \mid \Delta^{+}(\mu) X \in s_{\alpha}\left(\Delta^{+}(\lambda)\right)\right\},
$$

and since $\alpha / \lambda \in \Gamma$, we get

$$
s_{\alpha}\left(\Delta^{+}(\lambda)\right)=s_{(\alpha / \lambda)^{-}}
$$

We deduce that if $\alpha / \lambda \in \Gamma$,

$$
\left[\Delta^{+}, \Delta^{+}\right]=s_{(\alpha / \lambda)^{-}}\left(\Delta^{+}(\mu)\right) .
$$

Then, from Corollary 2.9, if $\alpha / \lambda \in \Gamma$ and $(\alpha / \lambda)^{-} / \mu=\left(\alpha_{n-1} / \lambda_{n-1} \mu_{n}\right)_{n} \in \Gamma$,

$$
s_{(\alpha / \lambda)^{-}}\left(\Delta^{+}(\mu)\right)=s_{\left((\alpha / \lambda)^{-} / \mu\right)^{-}}=s_{\left(\alpha_{n-2} / \lambda_{n-2} \mu_{n-1}\right)_{n}} .
$$

(vi) We have

$$
C^{+}(\lambda)(|C(\mu) X|) \in s_{\alpha} \Longleftrightarrow C(\mu) X \in w_{\alpha}^{+1}(\lambda),
$$

and since $\alpha \in \Gamma^{+}$, we have $w_{\alpha}^{+1}(\lambda)=s_{\alpha \lambda}$. Then for $\alpha \lambda \mu \in \Gamma, X \in\left[C^{+}, C\right]$ if and only if

$$
X \in \Delta(\mu) s_{\alpha \lambda}=s_{(\alpha \lambda \mu)}
$$

(vii) The condition $C^{+}(\lambda)(|\Delta(\mu) X|) \in s_{\alpha}$ is equivalent to

$$
\Delta(\mu) X \in w_{\alpha}^{+1}(\lambda),
$$

and since $\alpha \in \Gamma^{+}$, we have $w_{\alpha}^{+1}(\lambda)=s_{\alpha \lambda}$. Thus

$$
X \in s_{\alpha \lambda}(\Delta(\mu))=D_{1 / \mu} \Sigma s_{\alpha \lambda}=s_{(\alpha(\lambda / \mu))},
$$

since $\alpha \lambda \in \Gamma$. So (vii) holds. 
(viii) First, we have

$$
\left[C^{+}, \Delta^{+}\right]=\left\{X \mid \Delta^{+}(\mu) X \in w_{\alpha}^{+1}(\lambda)\right\},
$$

and the condition $\alpha \in \Gamma^{+}$implies that $w_{\alpha}^{+1}(\lambda)=s_{\alpha \lambda}$. Thus

$$
\left[C^{+}, \Delta^{+}\right]=\left\{X \mid \Delta^{+}(\mu) X \in s_{\alpha \lambda}\right\}=s_{\alpha \lambda}\left(\Delta^{+}(\mu)\right),
$$

and we conclude since

$$
s_{\alpha \lambda}\left(\Delta^{+}(\mu)\right)=s_{(\alpha \lambda / \mu)^{-}} \text {for } \frac{\alpha \lambda}{\mu} \in \Gamma .
$$

(ix) If $\alpha \in \Gamma^{+}$,

$$
\left[C^{+}, C^{+}\right]=\left\{X \mid C^{+}(\mu) X \in w_{\alpha}^{+1}(\lambda)=s_{\alpha \lambda}\right\}=w_{\alpha \lambda}^{+1}(\mu) .
$$

We conclude that $w_{\alpha \lambda}^{+1}(\mu)=s_{(\alpha \lambda \mu)}$, since $\alpha \lambda \in \Gamma^{+}$.

REMARK 3.4. Note that in Theorem 3.3, we have $\left[A_{1}, A_{2}\right]=s_{\alpha}\left(A_{1} A_{2}\right)=\left(s_{\alpha}\left(A_{1}\right)\right)_{A_{2}}$ for $A_{1} \in\left\{\Delta(\lambda), \Delta^{+}(\lambda), C(\lambda), C^{+}(\lambda)\right\}$ and $A_{2} \in\left\{\Delta(\mu), \Delta^{+}(\mu), C(\mu), C^{+}(\mu)\right\}$. For instance, we have

$$
[\Delta, C]=\left\{X \mid\left(\frac{\lambda_{n}}{\mu_{n}}-\frac{\lambda_{n-1}}{\mu_{n-1}}\right) \sum_{i=1}^{n-1} x_{i}+\frac{\lambda_{n}}{\mu_{n}} x_{n}=\alpha_{n} O(1)\right\} \quad \text { for } \frac{\alpha \mu}{\lambda} \in \Gamma .
$$

Similarly, under the corresponding conditions given in Theorems 3.1 and 3.3, we get

$$
\begin{aligned}
{[\Delta, \Delta] } & =\left\{X \mid-\lambda_{n-1} \mu_{n-2} x_{n-2}+\mu_{n-1}\left(\lambda_{n}+\lambda_{n-1}\right) x_{n-1}-\lambda_{n} \mu_{n} x_{n}=\alpha_{n} O(1)\right\}, \\
{\left[\Delta, C^{+}\right] } & =\left\{X \mid \frac{\lambda_{n}}{\mu_{n}} x_{n}+\left(\lambda_{n}-\lambda_{n-1}\right) \sum_{m=n-1}^{\infty} \frac{x_{m}}{\mu_{m}}=\alpha_{n} O(1)\right\}, \\
{\left[\Delta, \Delta^{+}\right] } & =\left\{X \mid-\lambda_{n-1} \mu_{n-1} x_{n-1}+\mu_{n}\left(\lambda_{n}+\lambda_{n-1}\right) x_{n}-\lambda_{n} \mu_{n+1} x_{n+1}=\alpha_{n} O(1)\right\}, \\
{\left[\Delta^{+}, \Delta\right] } & =\left\{X \mid-\lambda_{n} \mu_{n-1} x_{n-1}+\left(\lambda_{n}+\lambda_{n+1}\right) \mu_{n} x_{n}-\lambda_{n+1} \mu_{n+1} x_{n+1}=\alpha_{n} O(1)\right\} .
\end{aligned}
$$

\section{REFERENCES}

[1] B. de Malafosse, Systèmes linéaires infinis admettant une infinité de solutions [Infinite linear systems admitting an infinity of solutions], Atti Accad. Peloritana Pericolanti Cl. Sci. Fis. Mat. Natur. 65 (1988), 49-59 (French).

[2] - Some properties of the Cesàro operator in the space $s_{r}$, Commun. Fac. Sci. Univ. Ank. Ser. A1 Math. Stat. 48 (1999), no. 1-2, 53-71.

[3] _ Bases in sequence spaces and expansion of a function in a series of power series, Mat. Vesnik 52 (2000), no. 3-4, 99-112.

[4] _ Application of the sum of operators in the commutative case to the infinite matrix theory, Soochow J. Math. 27 (2001), no. 4, 405-421.

[5] _ Properties of some sets of sequences and application to the spaces of bounded difference sequences of order $\mu$, Hokkaido Math. J. 31 (2002), no. 2, 283-299.

[6] _ Recent results in the infinite matrix theory, and application to Hill equation, Demonstratio Math. 35 (2002), no. 1, 11-26. 
[7] _ Variation of an element in the matrix of the first difference operator and matrix transformations, Novi Sad J. Math. 32 (2002), no. 1, 141-158.

[8] _ On some BK spaces, Int. J. Math. Math. Sci. 2003 (2003), no. 28, 1783-1801.

[9] B. de Malafosse and E. Malkowsky, Sequence spaces and inverse of an infinite matrix, Rend. Circ. Mat. Palermo (2) 51 (2002), no. 2, 277-294.

[10] R. Labbas and B. de Malafosse, On some Banach algebra of infinite matrices and applications, Demonstratio Math. 31 (1998), no. 1, 153-168.

[11] I. J. Maddox, Infinite Matrices of Operators, Lecture Notes in Mathematics, vol. 786, Springer-Verlag, Berlin, 1980.

[12] E. Malkowsky, The continuous duals of the spaces $c_{0}(\Lambda)$ and $c(\Lambda)$ for exponentially bounded sequences $\Lambda$, Acta Sci. Math. (Szeged) 61 (1995), no. 1-4, 241-250.

[13] _ Linear operators in certain BK spaces, Approximation Theory and Function Series (Budapest, 1995), Bolyai Soc. Math. Stud., vol. 5, János Bolyai Mathematical Society, Budapest, 1996, pp. 259-273.

[14] F. Móricz, On $\Lambda$-strong convergence of numerical sequences and Fourier series, Acta Math. Hungar. 54 (1989), no. 3-4, 319-327.

[15] A. Wilansky, Summability through Functional Analysis, North-Holland Mathematics Studies, vol. 85, North-Holland Publishing, Amsterdam, 1984.

Bruno de Malafosse: Laboratoire de Mathématiques Appliquées du Havre (LMAH), Université du Havre, Institut Universitaire de Technologie du Havre, 76610 Le Havre, France

E-mail address: bdema 1 af@wanadoo.fr 


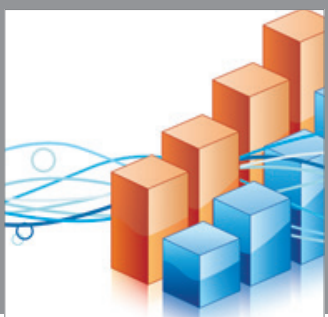

Advances in

Operations Research

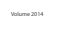

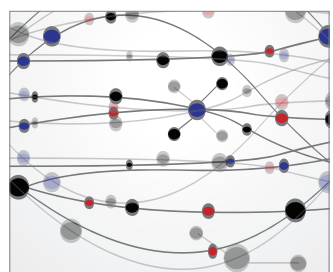

\section{The Scientific} World Journal
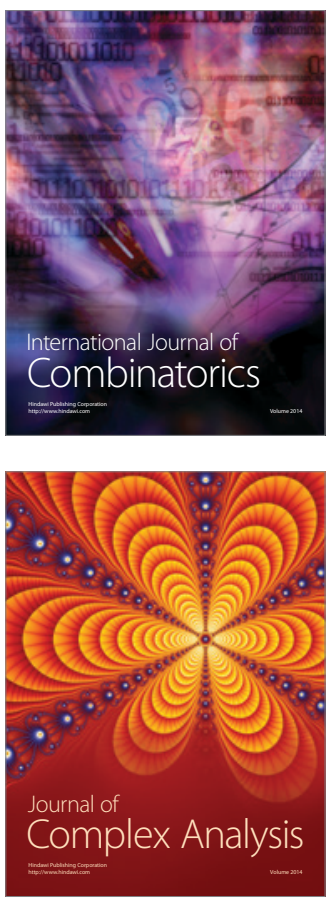

International Journal of

Mathematics and

Mathematical

Sciences
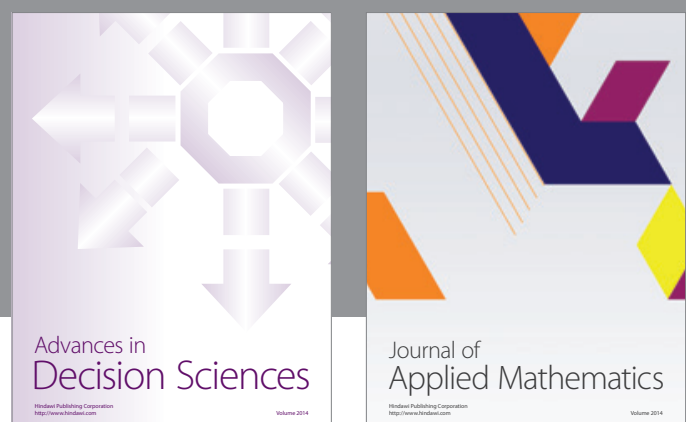

Journal of

Applied Mathematics
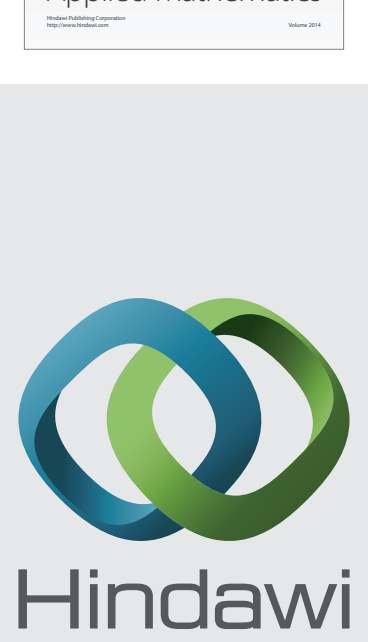

Submit your manuscripts at http://www.hindawi.com
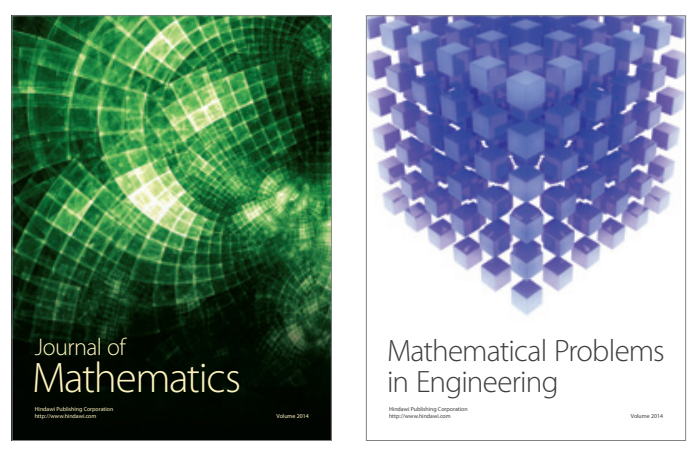

Mathematical Problems in Engineering
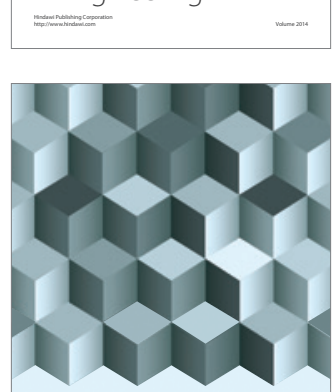

Journal of

Function Spaces
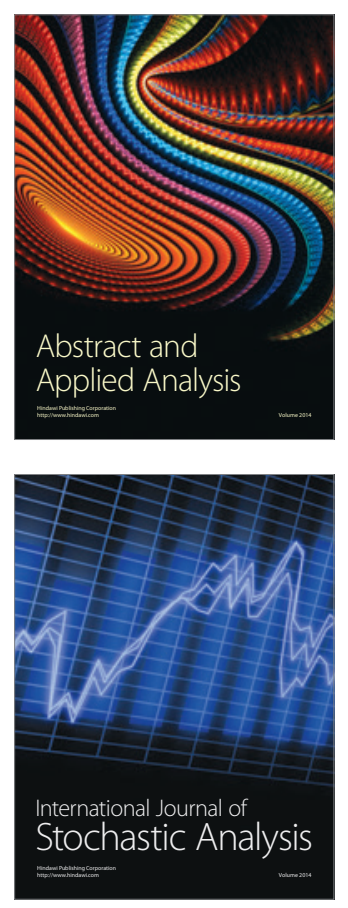

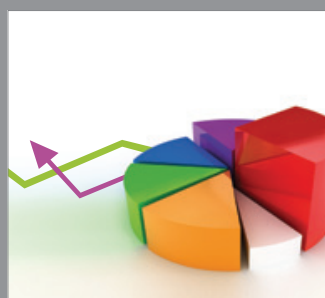

ournal of

Probability and Statistics

Promensencen
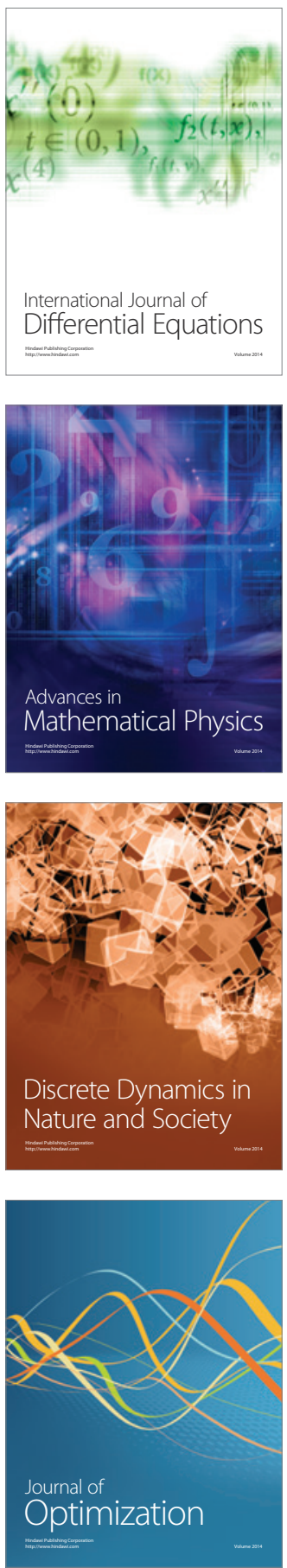